Background The clinical spectrum and associated factors of cerebral palsy may differ between developing and developed countries. Aim To evaluate the predisposing factors, clinical spectrum, and associated problems of cerebral palsy (CP) in children.

Settingand design In this retrospective study patient data were extracted from file records in our center for early diagnosis of childhood disabilities.

Patients and Methods Our study population included one hundred and twenty two children with age range from 7 months to 17 years. Patients were followed and reviewed in a 32 months period from September 2007 to April 2010.

Simple statistical analysis was used for percentage calculation. Results and Conclusions Spastic type was the predominant $(82.7 \%)$, with quadriplegic subtype being the most common (34.4\%). The other types were choreoathetoide (8.2\%) mixed type $(6.6 \%)$ and ataxic $(2.5 \%)$ being the least. Speech delay was the most common associated problem (71.3\%) followed by mental retardation $(61.5 \%)$, seizures $(35.2 \%)$, hearing problems (26.2), and autism (4.9\%) being the least. The clinical spectrum of CP in our country may differ from that reported from the western countries. Prospective studies are needed to evaluate the clinical spectrum and predisposing factors in Jordan.

\section{FUNCTIONAL PROGNOSIS IN OBSTETRICAL BRACHIAL PLEXUS PALSY}

doi:10.1136/archdischild-2012-302724.1520

'V Toopchizadeh, ${ }^{2} \mathrm{M}$ Barzegar. 'Physical Medicine and Rehabilitation, Tabriz Children Hospital Physical Mediane and Rehabilitation Research Ceter, Tabriz Median Sciences University; ${ }^{2}$ Pediatrics, Tabriz Children Hospital, Tabriz, Iran

Obstetrical brachial plexus palsy (OBPP) is a complication of difficult delivery and resulted from excessive traction on the brachial plexus during delivery. Erb palsy, klumpke paralysis and panplexus palsy reported in $46 \%, 0.6 \%$ and $20 \%$ of patients, respectively. Unilateral injury is more common than bilateral injury. Risk factors include macrosomia, multiparity, prior delivery of a child with OBPP, breech delivery shoulder dystocia, vacium and forceps assisted delivery and excessive maternal weight gain. The recovery rate is usually reported to be between 80 and $90 \%$. Management based on medical and surgical interventions. We evaluated 42 children with OBPP. Of them, we could follow only 28 cases during two years. Most of the patients were females. Right side palsy was more prevalent than left side palsy. Vaginal delivery without forceps was the most mode of delivery. Vertex was the most common presentation. Most of the patients were term. The mean weight of the birth was $3.8 \mathrm{~kg}$. Erb palsy and pan-plexus palsy consisted of $71.4 \%$ and $28.6 \%$ of lesions, Poor to moderate recovery occurred in 13 cases. good to complete (expected) recovery occurred in 15 cases.

\section{PAEDIATRIC NARCOLEPSY: A VIDEO SERIES}

doi:10.1136/archdischild-2012-302724.1521

'DK Gandhi, 'S Agrawal, 'L Notghi, 'E Wassmer. 'Paediatric Neurology; '2Paediatric Neurophysiology, Birmingham Children's Hospital, Birmingham, UK

Introduction Narcolepsy often begins in childhood (30\% of cases) but is infrequently recognized early in the course; only around $4 \%$ are diagnosed before age 15 . There have been many reports linking H1N1 and narcolepsy. We present a video series of 4 patients that highlight the diagnostic dilemma in paediatric cases as well as association with H1N1 flu.

Case Reports 4 children (5-11 years) were referred to the Neurologists for excessive daytime sleepiness. Disturbed sleep and cataplexy developed soon after. Immunosuppressive therapy was attempted with no response in one child with rapid progression. Cataplexy initially was focal or partial and progressed to the classical description. The Multiple Sleep Latency Test (MSLT) was grossly abnormal in all them. Hypocretin was absent in the one child investigated. Autoimmune screen was negative in all. One child was treated for $\mathrm{H} 1 \mathrm{~N} 1$ and 2 had the $\mathrm{H} 1 \mathrm{~N} 1$ vaccine prior to onset of symptoms.

Conclusion The key to early diagnosis and treatment is recognition of the condition. The videos highlight the facial features and other symptoms that are clues to narcolepsy (Pentad of narcolepsy). Rapid progression is possible related to $\mathrm{H} 1 \mathrm{~N} 1$ exposure.

\section{CLINICAL STUDY OF CHINESE MASSAGE COMBINED WITH MUSIC THERAPY IN TREATMENT OF CEREBRAL PALSY}

doi:10.1136/archdischild-2012-302724.1522

Z Liu. Cerebral Palsy Rehabilitation Centre of Nanhai Hospital for Women and Children, Affiliated to Guangzhou University of Chinese Medicine Guangdong China, Foshan, China

Objective Clinical observation of traditional chinese massage combined with music therapy to improve lower limbs' range of motion (ROM) and to reduce score of composite spasticity scale (CSS) in children with cerebral palsy (CP).

Methods Traditional chinese massage was performed to children with CP, such as to attack vital points of DU meridian, to tonify qi of kidney and slpeen, pinching massage, footplate massage, and segmental massage. Background music was played at the same time. Course of treatment was 30 days. ROM of ankle-joint and hip-joint, score of CSS was measures before and after the treatment.

Results 286 children with CP paticipated. 106 cases showed significant effect (37.40\%), 172 cases showed progress (59.16\%), and 8 cases had no-effect $(2.84 \%)$. Significant improvement were found after treatment for ROM of ankle-joint (before: 105.64 \pm 12.66 ; after:

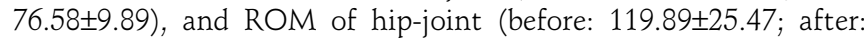
158.99 \pm 18.66$)(\mathrm{P}<0.01)$. Score of CSS was significantly lower after treatment (before: 12.39 \pm 2.56 ; after: $7.069 \pm 2.97)(\mathrm{P}<0.01)$.

Conclusion Traditional chinese massage combined music therapy can regulate qi and blood, solute muslce contracture and spasm, so as to rectify scissors gait and talipes, to improve gross motor function of children with CP. It is a therapy of convenience, economy and benefit.

\section{SCALP ACUPUNCTURE THERAPY OF CHILDREN WITH AUTISM}

doi:10.1136/archdischild-2012-302724.1523

Z Liu. Cerebral Palsy Rehabilitation Centre of Nanhai Hospital for Women and Children Affiliated to Guangzhou University of Chinese Medicine Guangdong China, Foshan, China

Objective Assessing the efficacy scalp acupuncture therapy for autism of children.

Methods We selected the 70 cases of autism children followed the random number method to divide the cases scalp acupuncture therapy group (30 cases) and structured control group (40 cases). The treated group with activating brain and opening orifices scalp acupuncture therapy, nordoff-robbins therapy and structured teaching, the control group with Nordoff-Robbins therapy and. We used the Clancy Autism Behavior Scale, Childhood Autism Behavior Scale, Autism Behavior Checklist, Gesell Development scale to assess the result before and after treatment, and adopted the blind method to evaluate the results.

\section{Results}

1. In the same treatment time, the therapy group is better than the control group.

2. Comparing among the treatment group, the older group and younger group, there have significant difference in $A B C$ score, CARS score and social adjustment score. 